\title{
Changes in Floquet State Structure at Avoided Crossings: Delocalization and Harmonic Generation
}

\author{
T. Timberlake and L. E. Reichl \\ Center for Studies in Statistical Mechanics and Complex Systems \\ The University of Texas at Austin \\ Austin, Texas 78712
}

(May 31, 2021)

\begin{abstract}
Avoided crossings are common in the quasienergy spectra of strongly driven nonlinear quantum wells. In this paper we examine the sinusoidally driven particle in a square potential well to show that avoided crossings can alter the structure of Floquet states in this system. Two types of avoided crossings are identified: one type leads only to temporary changes (as a function of driving field strength) in Floquet state structure while the second type can lead to permanent delocalization of the Floquet states. Radiation spectra from these latter states show a significant increase in high harmonic generation as the system passes through the avoided crossing.
\end{abstract}

42.50.Hz,05.45.+b,42.65.Ky

\section{INTRODUCTION}

Quantum systems whose classical counterparts are chaotic have received much attention in recent years [1]. One quantum signature of the classical transition to chaos is the appearance of avoided crossings in the spectrum of the quantum system. Avoided crossings occur when the curves of two quantum eigenvalues, as a function of some nonlinearity parameter, come near to crossing but then repel each other [2]. As the classical system becomes increasingly chaotic, the number of avoided crossings in the quantum spectrum increases [3] 5 . This repulsion of eigenvalue curves leads to a change from Poisson to random-matrix statistics in the eigenvalue spacings. These avoided crossings also play an important role in dynamical tunneling [6],7].

Another common feature of these classically chaotic quantum systems is that their eigenstates often show nonclassical localization. The probability density for these localized states remains in a small region of the phase space, even though there is no classical barrier to prevent them from spreading. Even systems with globally chaotic classical motion can have localized quantum eigenstates. This phenomenon was originally termed scarring $[8]$ and it was found that the localization tends to occur near the paths of classical periodic orbits.

Understanding localization is particularly important in light of some recent discoveries in the field of atomic physics. Experiments on atoms illuminated by an intense laser field show that the atoms can emit photons whose frequencies are many times the frequency of the incident laser [9.10]. Recent theoretical work has shown that this high harmonic generation is related to delocalization and chaos [11, 12]. Theoretical work has also shown that in some cases the ionization rate of these atoms may actually decrease as the intensity of the laser is increased [13, 14]. Some experimental evidence for this stabilization has been found [15]. Localization plays a major role in stabilizing these atoms against ionization [16]. Therefore we would like to have a better understanding of how localized eigenstates are created and destroyed in these systems, and what relationship this has to the other phenomena of "chaotic" quantum systems.

In this paper we will show that avoided crossings have a major impact on localization. A connection between level repulsion and the creation of extended (delocalized) states has already been found [17,18]. However, these studies looked at statistical properties of the system as a whole. Here we will concentrate on the changes in the structure of the quantum eigenstates at a single avoided crossing. We identify two distinct types of avoided crossings which have different effects on eigenstate structure. One type of crossing produces significant changes in the eigenstates only for the parameter values at which the avoided crossing actually occurs. The second type results in structural changes that persist for parameter values that are beyond the avoided crossing. Finally, we will investigate what impact these structural changes have on the radiation spectrum of the system.

\section{CLASSICAL AND QUANTUM DYNAMICS OF THE DRIVEN SQUARE WELL}

The model we will use to study these phenomena is the sinusoidally driven square well. We choose this model because of its simplicity and its connection with experimental work in solid-state physics. The driven square well 
serves as a highly simplified model for experiments involving electrons confined in GaAs/AlGaAs wells and subjected to intense far-infrared radiation [19,20]. This model is also advantageous because it has been the subject of many theoretical studies, both classical and quantum. Its basic properties are well understood, allowing us to focus on the particular phenomenon we are interested in.

\section{A. Classical Dynamics}

The Hamiltonian for the driven square well is:

$$
\tilde{H}=\frac{\tilde{p}^{2}}{2 m}+\tilde{\epsilon} \tilde{x} \cos \tilde{\omega}_{0} \tilde{t},|\tilde{x}| \leq a,
$$

where $m$ is the mass, $\tilde{p}$ is the momentum, and $\tilde{x}$ is the position of the particle. The width of the square well is $2 a$. The driving field has amplitude $\tilde{\epsilon}$ and frequency $\tilde{\omega_{0}}$, with $\tilde{t}$ as the time coordinate. This Hamiltonian can be made dimensionless using the scaling transformation introduced in [21], where $\tilde{H}=H c, \tilde{x}=x a, \tilde{p}=p \sqrt{2 m c}, \tilde{\epsilon}=\epsilon c / a$, $\tilde{t}=t a \sqrt{2 m / c}$, and $\tilde{\omega}_{0}=\left(\omega_{0} / a\right) \sqrt{c /(2 m)}$. The parameter $c$ is a new unit of energy in terms of which the scaled Hamiltonian will be expressed. The scaled Hamiltonian (in units of $c$ ) is:

$$
H=p^{2}+\epsilon x \cos \omega_{0} t,|x| \leq 1
$$

where all quantities are now dimensionless.

Note that $\epsilon$ and $\omega_{0}$ are not independent parameters, since the transformation $\left(\omega_{0}, \epsilon\right) \rightarrow\left(\omega_{0} \sqrt{c}, \epsilon c\right)$ produces the same dynamics (with a rescaling of the energy unit c). Because of this scaling law we can choose an arbitrary $\omega_{0}$, study the dynamics as a function of $\epsilon$, and effectively analyze the dynamics for any set of $\left(\omega_{0}, \epsilon\right)$. In this paper we choose $\omega_{0}=80$.

The driving field induces a series of nonlinear resonances of odd order in the square well system, with higher order resonances occurring at lower energies [21,22]. This means that the $N=1$ primary resonance sits at a higher energy than all of the other resonances. As the strength of the driving field is increased the resonances overlap and the dynamics in the region of overlap becomes chaotic. At very high field strengths all higher order resonances have been destroyed and only the $N=1$ resonance remains. As the field strength is increased still further, even this $N=1$ resonance begins to disappear. However, since there are no resonances at higher energies the region of chaos remains bounded from above by regular motion. This bounded chaos can be seen clearly in strobe plots of the classical motion of this system. Figure 1 shows strobe plots of this system for $\epsilon=175$ and 750 . The coordinates for these plots are the action-angle variables of the undriven square well, defined by $J=2|p| / \pi$ and $\theta= \pm \pi(x+1) / 2$ where all quantities are dimensionless because of the above scaling.

\section{B. Quantum Dynamics and Floquet Theory}

The Schrödinger equation for a driven particle in an infinite square well is given by

$$
i \hbar \frac{\partial}{\partial \tilde{t}}|\psi(\tilde{t})\rangle=-\frac{\hbar^{2}}{2 m} \frac{\partial^{2}}{\partial \tilde{x}^{2}}+\tilde{\epsilon} \tilde{x} \cos \left(\tilde{\omega_{0}} \tilde{t}\right)
$$

where $|\tilde{x}| \leq a$. All parameters are defined as in the classical Hamiltonian above. The transformation to dimensionless coordinates is identical to that used for the classical Hamiltonian, except that instead of scaling $\tilde{p}$ we must scale $\hbar$. The resulting dimensionless equation is

$$
i \kappa \frac{\partial}{\partial t}|\psi(t)\rangle=\left(-\kappa^{2} \frac{\partial^{2}}{\partial x^{2}}+\epsilon x \cos \left(\omega_{0} t\right)\right)|\psi(t)\rangle
$$

where energy is measured in units of $c$ (as in the classical case) and $\kappa=\hbar /(a \sqrt{2 m c})$. Note that in the quantum system there are three parameters: $\epsilon, \omega_{0}$, and $\kappa$. Only two of these parameters are independent, so the full dynamics of this system can be studied by varying two parameters. In this paper we will only vary one of the parameters, $\epsilon$, and we set $\kappa=1$ and $\omega_{0}=80$ as above. The effect of varying $\kappa$ is left for future study.

This quantum system is periodic in time and can be described in terms of Floquet eigenstates [23, 24], which are simply eigenstates of the one-period time evolution operator. Since the time evolution operator is unitary for this system (there is no ionization) all of the eigenvalues of the operator have modulus 1. Thus the Floquet states satisfy 


$$
\hat{U}(T)\left|\Omega_{\alpha}\right\rangle=e^{-i \Omega_{\alpha} T / \kappa}\left|\Omega_{\alpha}\right\rangle,
$$

where $\Omega_{\alpha}$ is the Floquet eigenvalue (or quasienergy) and $T$ is the period of the driving field. It is apparent from the above equation that the quasienergy is only defined modulo $\omega_{0}=2 \pi / T$ (with $\kappa=1$ ).

The Floquet states can be computed numerically by first calculating the matrix for the operator $\hat{U}(T)$ in a basis of unperturbed square well eigenstates. A numerical diagonalization of this matrix produces the eigenvalues and eigenvectors (in the unperturbed basis). Since there is little coupling between states in the regular region and states in the region of bounded chaos, a basis that extends into the regular region will give an accurate description of all states associated with the chaotic region [25]. In our calculations we use a basis of 80 eigenstates. With $\kappa=1$ this basis extends far into the regular region for all values of $\epsilon$ that we will consider here.

\section{AVOIDED CROSSINGS}

We wish to study the quantum dynamics that takes place near an avoided crossing (AC) in the spectrum of quasienergies for this system. Our first step then is to locate some avoided crossings. Figure 2 shows the $\left(\bmod \omega_{0}\right)$ spectrum of the 40 lowest quasienergies as a function of $\epsilon$. As mentioned in Section I, there is a close connection between the onset of ACs and chaos in the classical system. Figure 2 shows that avoided crossings begin to appear between $\epsilon=100$ and $\epsilon=200$, and by $\epsilon=800$ the spectrum is dominated by avoided crossings. This compares well with the growth of the chaotic region in the classical phase space between these two values of $\epsilon$ (see Fig. 1).

It is important to note that there are many places, particularly at small values of $\epsilon$, where the quasienergy curves actually cross. This happens when the two states associated with the curves belong to different (uncoupled) sectors of the Hilbert space and transitions between these states are forbidden 2, 26, 27]. By different sectors of Hilbert space we mean that the two states belong to different blocks of a block-diagonal Hamiltonian, indicating that they belong to different symmetry groups. When $\epsilon$ is small these "apparent crossings" are quite common, but at large $\epsilon$ there are few apparent crossings. The reason for this is that the spread of chaos is due to the breaking of the same symmetries that prevent coupling of certain states.

There are many strong ties between avoided crossings and chaos, beyond the observed increase of ACs as the classical system becomes chaotic. Successive avoided crossings are responsible for the transition to a random-matrix distribution [4], a property which has long been associated with chaos. In fact, there is a strong correlation between the overlap of these successive ACs and the fraction of the classical phase space which is chaotic [5]. Other studies have shown that ACs occur only when the two states can tunnel through any Kolmogorov-'Arnold-Moser (KAM) tori that lie between them in the phase space [28]. This means that the states involved in avoided crossings tend to lie in regions of the phase space where the KAM tori have been strongly distorted or destroyed altogether 19 .

By studying Fig. 2 we can formulate a general picture of what is happening to the quasienergy curves of this system as $\epsilon$ is increased. At low $\epsilon$ there are no avoided crossings and most of the quasienergy curves maintain a constant slope. The only curvature here is in the set of curves that look like the characteristic curves of the Mathieu equation [29]. The states associated with these curves are becoming trapped in the pendulum-like $N=1$ primary resonance that forms as $\epsilon$ increases from 0 . The curve that looks like the ground state of the Mathieu equation is connected to the $\mathrm{n}=16$ square well state at $\epsilon=0$. Note that $J=16$ is the exact position of the $N=1$ resonance in the strobe plots of Fig. 1. At higher values of $\epsilon$ ACs begin to appear. At the avoided crossing itself there is a significant change in the slope of the quasienergy curves, but at these moderate values of $\epsilon$ the AC seems to result only in an exchange of slopes between the two curves. At the highest values of $\epsilon$ shown in Fig. 2 the avoided crossings result in dramatic changes in the slopes of the quasienergy curves, not just an exchange of slope. One may also note that the Mathieu curves are no longer identifiable at these values of $\epsilon$. At these high values of $\epsilon$ the $N=1$ resonance has become highly distorted and is beginning to disappear into the chaotic sea (see Fig. 1 p). Thus we see that there is a strong connection between changes in the classical phase space and changes in the quasienergy spectrum.

\section{CHANGES IN FLOQUET STATE STRUCTURE AT AN AVOIDED CROSSING}

This connection between chaos and quasienergy curves is interesting, but it is not entirely clear. For one thing, only the connection between very large changes in the classical phase space and correspondingly large changes in the quasienergy spectrum has been established. We would like to study the changes that take place at a single avoided crossing. Additionally, we would like to see changes in the structure of the Floquet states, rather than changes in the

quasienergy curves. To see these structural changes we must construct a quantum mechanical "phase space". We can then monitor the changes in the "phase space" as the value of $\epsilon$ moves through an avoided crossing. 


\section{A. Visualization of Quantum Phase Space}

Visualization of the quantum mechanical "phase space" requires the construction of a phase space probability density for the various quantum eigenstates. The uncertainty principle prevents the construction of a true phase space probability density in the classical sense, but it is possible to construct a quasiprobability density that is positive definite and gives a coarse-grained picture of the distribution of the quantum state in phase space. This quasiprobability density is the Husimi distribution [30,31]. To construct the Husimi distribution of a given quantum state one simply calculates the overlap between the given state and a coherent state centered on a particular point $\left(x_{0}, p_{0}\right)$ in phase space. The wavefunction of the coherent state in configuration space is 32

$$
\left\langle x \mid x_{0}, p_{0}\right\rangle=\left(\frac{1}{\sigma^{2} \pi}\right)^{1 / 4} \exp \left(-\frac{\left(x-x_{0}\right)^{2}}{2 \sigma^{2}}+\frac{i p_{0}\left(x-x_{0}\right)}{\hbar}\right)
$$

where $\sigma$ is a squeezing parameter that determines the relative widths of the coherent state in the $x$ and $p$ directions. Calculating $\left|\left\langle\psi \mid x_{0}, p_{0}\right\rangle\right|^{2}$ for a grid of phase space points will produce a quasiprobability distribution that can be easily viewed as a contour plot. Figures 6 and 6 show Husimi distributions for several Floquet states in this system.

Now we can use the Husimi distribution to visualize changes in the structure of the quantum phase space that take place at an AC. We will examine the Husimi distributions of Floquet states at values of $\epsilon$ slightly less than, slightly greater than, and at the value at which that state undergoes an AC. This will allow us to determine what changes occur at the $\mathrm{AC}$ and to what extent these changes survive at higher values of $\epsilon$.

A close inspection of Fig. 22 reveals that not all avoided crossings look the same. As discussed in Sec. III there are crossings where the curves simply exchange slopes and crossings where the slopes change. We will refer to the crossings that exchange slopes as sharp ACs. The states involved in such an AC are weakly coupled and usually lie in different regions of the phase space (inside a resonance and in the chaotic region, for instance). The other type, broad ACs, involve strongly coupled states that usually reside in the same region of phase space. In fact, most broad ACs occur between states that are associated with the region of chaos.

\section{B. Sharp Crossing}

Figure 3 is a detail from Fig. 2 that focuses on a sharp avoided crossing at $\epsilon \approx 175$. The two curves that participate in the avoided crossing are labeled A and B. In Fig. 1 we show the Husimi distributions of the two Floquet states at $\epsilon=170,175.5$, and 180. At $\epsilon=170$ we can see that state $\mathrm{A}$ is contained within the $\mathrm{n}=1$ primary resonance (see Fig. 17a for a picture of the classical dynamics near this field strength) while state B lies in the low-energy chaotic region. At $\epsilon=175.5$, the center of the avoided crossing, the Husimi distributions for both states appear to be mixtures of the states shown for $\epsilon=170$. Clearly the AC has a dramatic impact on the structure of these states at this particular value of $\epsilon$. However, the Husimi distributions at $\epsilon=180$ show that these changes do not persist at higher field strengths. The two states simply exchange their structure, so that the net effect is a relabeling of the Floquet states. So away from the AC itself the overall structure of the quantum phase space is unchanged.

These dynamics can be understood quite well using a two-level approach [33]. These sharp ACs really only involve the two Floquet states whose curves nearly come together. The rest of the Hilbert space has very little influence on the dynamics of these two states. Without any contribution to the dynamics from other states, these two levels can only exchange their structure. These types of crossings play an important role in tunneling, since a state originally confined to a resonance has become a chaotic state after the AC [6], but they do not play a significant role in altering the structure of the quantum phase space.

\section{Broad Crossing}

In a broad AC several states make significant contributions to the dynamics. A particularly striking example of this is shown in Fig. 河, where two ACs (at $\epsilon \approx 750$ and $\epsilon \approx 765$ ) actually overlap. Here there are three states (labeled $\mathrm{C}, \mathrm{D}$, and E) that are strongly influencing each others' dynamics. There is no simple exchange of slopes between the curves in this AC. Hence, we might expect to find more interesting (and permanent) structural changes in this crossing than in the previously studied one. Figure 6 shows the effect of the avoided crossing on the structure of the three states that are involved. As in the sharp crossing there is a mixing of structures for values of $\epsilon$ that lie within the crossing region. However, in this case the changes do not disappear when we look at larger values of field strength. With this type of AC the states are not simply "relabeled", but undergo actual changes in their phase space 
structure. Particularly striking is the difference between Figs. 6 $\mathrm{cc}$ and $6 \mathrm{~h}$. These states would be identical if there was a complete exchange of structure as seen in the sharp AC. Instead, before the crossing state $\mathrm{E}$ is localized at very low energies, but after the crossing state D (which has exchanged most of its structure with state E) has spread into the higher energy portion of the region of chaos. The avoided crossing has delocalized this particular Floquet state. We find this result to be quite general, that broad ACs lead to permanent changes in the structure of Floquet states that tend to delocalize the states. Of course, since the region of chaos is bounded the states can only delocalize until they reach the boundaries of the chaos. At extremely high values of $\epsilon$, where nearly every "chaotic" state has undergone many broad avoided crossings, we find that all of these states are delocalized and fill the chaotic region [11].

\section{EFFECT OF STRUCTURAL CHANGES ON RADIATION SPECTRA}

Now that we have seen how avoided crossings can affect the structure of Floquet states we would now like to see how they effect an experimentally observable quantity, namely the radiation spectrum. In a prior work we found that the generation of high harmonics increased as the system, initially in a single Floquet state, passed through the avoided crossing [11]. However, this effect was caused by population transfer from the original Floquet state to the other state as the field strength was increased. This population transfer creates a superposition of two Floquet states that displays increased radiation at high frequencies (albeit shifted away from the harmonic frequencies). In this study we would like to focus on changes in the radiation spectrum that are caused by the changing structure of a single Floquet state. For this reason we will calculate radiation spectra by starting the system in a given Floquet state and maintaining a constant field strength for 128 cycles of the driving field. The expectation value of the acceleration for the state is calculated during this time interval. We then calculated the Fourier transform, $\xi(\omega)$, of this acceleration time series. The square modulus of the Fourier transform gives us the radiation spectrum. Because the Floquet states are periodic with period $2 \pi / \omega_{0}$ it is not truly necessary to calculate for 128 cycles of the driving field. However, this long integration time exposes numerical errors that might be hidden in a shorter calculation.

To study the effect of structural changes on radiation spectra we cannot simply study the spectrum of a single Floquet state for various values of $\epsilon$. Avoided crossings cause states to exchange structure, effectively relabeling the states. If true structural changes are to be distinguished from simple relabeling, one must account for this relabeling when comparing different spectra. At the midpoint of the avoided crossing this is nearly impossible to do, since the relabeling has not fully taken effect. For values of $\epsilon$ that are beyond the AC, the relabeling can easily be taken into account. Our procedure in the following is to calculate spectra for a state before the AC, the same state (on the same curve) at the midpoint of the AC, and the relabeled state (now on a different quasienergy curve) after the AC. This separates the changes in radiation spectrum that occur because of structural change in the Floquet state from the apparent changes that occur because the states have been relabeled.

\section{A. Sharp Crossing}

We first calculate radiation spectra for the states whose Husimi distributions are shown in Fig. 1(a,c, and f). The first two states are associated with the curve A in Fig. 3, while the third is associated with curve B. By changing from A to B after the avoided crossing we can account for the effects of relabeling as described above. The spectra are shown in Fig. 7. Between $\epsilon=170$ and $\epsilon=175.5$ there is a significant increase in the radiation at the highest harmonics (11-19). However, these harmonics have decreased at $\epsilon=180$. This increase and subsequent reduction is easier to see in Fig. 8, which shows the differences between the spectrum at $\epsilon=170$ and the spectra at $\epsilon=175.5$ and 180. This temporary increase in high harmonic generation is exactly what we expect from the temporary changes in the phase space structure seen in Fig. 1. At the midpoint of the AC both states are a mixture of the two states at $\epsilon=170$ and both are spread over a wider region of phase space. This leads to an increase in harmonic generation at this value of $\epsilon$. After the avoided crossing, however, these structural changes disappear (with the exception of the relabeling) and the harmonic generation subsides. Thus, sharp ACs only affect the radiation spectrum of a Floquet state for field strengths that lie within the crossing.

\section{B. Broad Crossing}

Now we investigate the radiation spectra for the states shown in Fig. 6(c, f, and h). Again we switch from state $\mathrm{E}$ to state $\mathrm{D}$ for values of $\epsilon$ that are beyond the avoided crossing, to account for the relabeling that takes place. Fig. 9 shows the spectra for these states. There is a steady increase in the radiation at the highest harmonics (31-45) as 
$\epsilon$ is increased. This is more easily seen in Fig. 10 which shows the differences between the spectra. The changes in the spectra are quite complicated, but there is clearly no reversal in the increase of high harmonics as was observed in the sharp crossing. This broad AC permanently increases the high harmonic generation. This is closely tied to the delocalization that is observed in Fig. 6, since the generation of high harmonics depends on the number of energy levels over which the Floquet state is spread [11].

\section{CONCLUSION}

We have seen here that avoided crossings can dramatically alter the phase space structure of Floquet states in a driven quantum system. Sharp ACs, involving states in separate sectors of the Hilbert space, produce structural changes that do not survive at higher field strengths. The only effect that remains at higher field strengths is a relabeling of the Floquet states. These crossings increase the HHG from a Floquet state at the crossing itself, but they do not lead to increased HHG at higher field strengths. Broad crossings, however, usually involve states in the same sector of the Hilbert space and often include effects from several states at once. These crossings can create persistent changes in the structure of the Floquet states and in the radiation spectra that these states produce. At field strengths beyond the avoided crossing the states will be less localized and the spectra will show stronger radiation at high harmonics.

The data on harmonic generation is interesting, as high harmonic generation has gained a lot of attention in recent years. But perhaps even more intriguing are the results that show how avoided crossings delocalize Floquet states. This has bearing on the important problem of the stabilization of atoms in intense laser fields. Theoretical studies have shown that this stabilization may result from the electron occupying a Floquet state that is localized in the phase space [16]. This localization prevents the electron from reaching the continuum and thus decreases the ionization rate. To better understand stabilization one must understand how localized Floquet states are created and destroyed. Our work indicates that avoided crossings play a key role in this process. It must be noted, however, that the model used in this paper does not allow ionization. To study stabilization this work must be extended to open quantum systems.

Although the primary motivation for this study was theoretical, these results may be experimentally observable. The infinite square well serves as a simple model for recent experiments on electron confinement in GaAs/AlGaAs quantum wells $[19,20]$. These experiments confine electrons in wells that vary in width from 50 to $1000 \AA$ and in depth from 200 to $300 \mathrm{meV}$. A well with a width of only $50 \AA$ and a depth of $300 \mathrm{meV}$ contains only a few bound states and therefore cannot be expected to produce the effects seen in this study. However, a well with a depth of $300 \mathrm{meV}$ and a width of $600 \AA$ contains about 50 bound states. The dynamics of such a well, driven by a far-infrared laser at low intensity, should be similar to the dynamics of our model. The parameters used in this paper correspond to a laser with a wavelength of $400 \mu \mathrm{m}$ and intensity of $10^{5} \mathrm{~W} / \mathrm{cm}^{2}$ striking a $600 \AA$ well with a depth of $300 \mathrm{meV}$. These parameters are well within the range accessible by recent experiments.

\section{ACKNOWLEDGMENTS}

The authors wish to thank the Welch Foundation Grant No. F-1051 and DOE Contract No. DE-FG03-94ER14465 for partial support of this work. We also thank NPACI and the University of Texas at Austin High Performance Computing Center for use of their computer facilities.

[1] L. E. Reichl, The Transition to Chaos In Conservative Classical Systems: Quantum Manifestations (Springer-Verlag, Berlin, 1983).

[2] J. von Neumann and E. Wigner, Phys. Z. 30, 467 (1929). [English translation in R. Knox and A. Gold, Symmetry in the Solid State (W. A. Benjamin, Inc., New York, 1964).]

[3] J. Zakrzewski and D. Delande, Phys. Rev. E 47, 1665 (1993).

[4] S.-J. Wang and S. Y. Chu, Phys. Rev. A 47, 3546 (1993).

[5] X. Yang and J. Burgdörfer, Phys. Rev. A 48, 83 (1993).

[6] M. Latka, P. Grigolini, and B. J. West, Phys. Rev. A 50, 1071 (1994).

[7] M. Latka, P. Grigolini, and B. J. West, Phys. Rev. E 50, 596 (1994).

[8] E. J. Heller, P. W. O'Connor, and J. Gehlen, Phys. Scr. 40, 354 (1989). 
[9] J. J. Macklin, J. D. Kmetec, and C. L. Gordon, Phys. Rev. Lett. 70, 766 (1993).

[10] Z. Chang, A. Rundquist, H. Wang, M. M. Murnane, and H. C. Kapteyn, Phys. Rev. Lett. 79, 2967 (1997).

[11] W. Chism, T. Timberlake, and L. E. Reichl, Phys. Rev. E 58, 1713 (1998).

[12] V. Averbukh and N. Moiseyev, Phys. Rev. A 51, 3911 (1995).

[13] M. Pont and M. Gavrila, Phys. Rev. Lett. 65, 2362 (1990).

[14] R. M. Potvliege and P. H. G. Smith, Phys. Rev. A 48, R46 (1993).

[15] M. P. de Boer, J. H. Hoogenraad, R. B. Vrijen, R. C. Constantinescu, L. D. Noordam, and H. G. Muller, Phys. Rev. A 50, 4085 (1994).

[16] B. Sundaram and R. V. Jensen, Phys. Rev. A 47, 1415 (1993).

[17] W. A. Lin and L. E. Reichl, Phys. Rev. A 40, 1055 (1989).

[18] J. Zakrzewski and D. Delande, Phys. Rev. E 47, 1650 (1993).

[19] B. Galdrikian, B. Birnir, and M. Sherwin, Phys. Lett. A 203, 319 (1995).

[20] B. Birnir, B. Galdrikian, R. Grauer, and M. Sherwin, Phys. Rev. B 47, 6795 (1993).

[21] W. A. Lin and L. E. Reichl, Physica D 19, 145 (1986).

[22] M. Fuka, J. K. McIver, W. Becker, M. Orszag, and R. Ramírez, Phys. Rev. E 51, 1935 (1995).

[23] J. H. Shirley, Phys. Rev. 138, B979 (1965).

[24] H. Sambe, Phys. Rev. A 39, 2203 (1973).

[25] H. P. Breuer and M. Holthaus, Z. Phys. D 11, 1 (1989).

[26] M. V. Berry and M. Wilkinson, Proc. R. Soc. Lond. A 392, 15 (1984).

[27] H. P. Breuer, K. Dietz, and M. Holthaus, Z. Phys. D 8, 349 (1988).

[28] J. Henkel and M. Holthaus, Phys. Rev. A 45, 1978 (1992).

[29] See Handbook of Mathematical Functions, Natl. Bur. Stand. Appl. Math. Ser. No. 55, edited by M. Abramowitz and I. A. Stegun (U. S. GPO, Washington, DC, 1968).

[30] K. Husimi, Proc. Phys. Math. Soc. Jpn. 22, 248 (1940).

[31] K. Takahashi, Prog. Theor. Phys. Suppl. 98, 109 (1989).

[32] B. Crespi, G. Perez, and S.-J. Chang, Phys. Rev. E 47, 986 (1993).

[33] Q. Jie, S.-J. Wang, and L.-F. Wei, Z. Phys. B 104, 373 (1997).

FIG. 1. Strobe plots of the classical dynamics of the driven square well at two field strengths. $J$ and $\theta$ are the dimensionless action-angle variables for the undriven square well. In (a) $\epsilon=174$ and the primary $N=1$ resonance is very prominent within the region of bounded chaos. In (b) $\epsilon=780$ and the $N=1$ resonance is distorted and occupies a smaller region of phase space than at $\epsilon=174$. The chaotic region is much larger at this higher field strength.

FIG. 2. Spectrum of quasienergies for the driven square well as a function of field strength. The first avoided crossings appear between $\epsilon=100$ and $\epsilon=200$. As $\epsilon$ is increased the number of avoided crossings increases rapidly so that by $\epsilon=800$ avoided crossings dominate the spectrum. Both $\Omega_{\alpha}$ and $\epsilon$ are dimensionless quantities.

FIG. 3. Detail from Figure 1 showing a sharp avoided crossing near $\epsilon=175.5$. The quasienergy curves involved in the avoided crossing are labeled $\mathrm{A}$ and $\mathrm{B}$.

FIG. 4. Husimi distributions of the Floquet states involved in the avoided crossing shown in Figure 3. The labels A and B indicate the quasienergy curve in Fig. 3 with which the state is associated. The $\epsilon$ values indicate the field strength at which the Floquet state was calculated. At $\epsilon=175.5$ (the center of the avoided crossing) both Floquet states are mixtures of the two states at $\epsilon=170$. By $\epsilon=180 \mathrm{~A}$ and $\mathrm{B}$ have exchanged their structure completely. Note that the coordinates for all of the Husimi plots are the dimensionless action-angle variables used in Fig. 1 .

FIG. 5. Detail of Figure 2 showing a pair of broad avoided crossings near $\epsilon=760$. The three quasienergy curves that are involved in the avoided crossing are labeled C, D, and E.

FIG. 6. Husimi distributions of the three Floquet states involved in the crossings shown in Figure 5. The labels C, D, and E indicate the quasienergy curve in Fig. 5 with which the state is associated. At $\epsilon=760$ all three states are mixtures of the states at $\epsilon=740$. By $\epsilon=780$ the states have exchanged most of their structure, but there are significant differences from the $\epsilon=740$ states. In particular, we expect the states (c) and (h) to have similar structure but instead find that (h) is much less localized than (c). 
FIG. 7. Radiation spectra generated by the Floquet states shown in Figure $1(\mathrm{a}, \mathrm{c}, \mathrm{e})$. There is a significant increase in harmonic generation from $\epsilon=170$ (a) to $\epsilon=175.5$ (b). However, by $\epsilon=180$ (c) this increase has disappeared. All spectra have been normalized so that they have the same power at the fundamental frequency $\omega_{0}$.

FIG. 8. Differences between spectra shown in Figure 7. The difference between Fig. Wb and Fig. 月a is shown in (a). The difference between $\mathbb{j}_{\mathrm{c}}$ and $\mathrm{J}_{\mathrm{a}}$ is shown in (b). The increase and subsequent decrease in harmonic generation is apparent.

FIG. 9. Radiation spectra generated by the Floquet states shown in Figure $6(\mathrm{c}, \mathrm{f}, \mathrm{h})$. There is a significant increase in harmonic generation from $\epsilon=740$ (a) to $\epsilon=760$ (b) and by $\epsilon=780$ the harmonic generation has increased even further. All spectra have been normalized so that they have the same power at the fundamental frequency $\omega$.

FIG. 10. Differences between spectra shown in Figure 8. The difference between Fig. Bb and Fig. 8a is shown in (a). The difference between $\mathrm{Gc}$ and $\mathrm{Ba}$ is shown in (b). The increase in harmonic generation seen in (a) clearly persists in (b). 
This figure "figure1.GIF" is available in "GIF" format from: http://arxiv.org/ps/quant-ph/9810014v1 
This figure "figure2.GIF" is available in "GIF" format from: http://arxiv.org/ps/quant-ph/9810014v1 
This figure "figure3.GIF" is available in "GIF" format from: http://arxiv.org/ps/quant-ph/9810014v1 
This figure "figure4.GIF" is available in "GIF" format from: http://arxiv.org/ps/quant-ph/9810014v1 
This figure "figure5.GIF" is available in "GIF" format from: http://arxiv.org/ps/quant-ph/9810014v1 
This figure "figure6.GIF" is available in "GIF" format from: http://arxiv.org/ps/quant-ph/9810014v1 
This figure "figure7.GIF" is available in "GIF" format from: http://arxiv.org/ps/quant-ph/9810014v1 
This figure "figure8.GIF" is available in "GIF" format from: http://arxiv.org/ps/quant-ph/9810014v1 
This figure "figure9.GIF" is available in "GIF" format from: http://arxiv.org/ps/quant-ph/9810014v1 
This figure "figure 10.GIF" is available in "GIF" format from: http://arxiv.org/ps/quant-ph/9810014v1 